\title{
Associação entre a expressão das proteínas p53 e Ki-67 e os achados clínico-patológicos em pacientes com carcinoma invasor do colo uterino
}

\author{
Association between p53 and Ki-67 expression and clinicopathologic features in patients with \\ carcinoma of the cervix
}

Agnaldo L. Silva-Filhoㄹ, Bernardo Nascentes Bruno², Lucas Barbosa da Silva ${ }^{3}$, Paulo Traiman ${ }^{4}$, João Gilberto de Castro e Silva ${ }^{5}$, Sérgio Augusto Triginelli ${ }^{6}$.

\section{RESUMO}

Objetivo: avaliar a associação da expressão das proteínas p53 e Ki-67 no tumor com achados clínico-patológicos em pacientes com carcinoma invasor de colo uterino. Métodos: foram estudadas amostras de tumor obtidas de 36 pacientes submetidas a histerectomia radical para tratamento de carcinoma invasor do colo uterino estádio IB (FIGO). Amostras do tumor foram fixadas em formol e incluídas em parafina. O material foi analisado pela histopatologia (hematoxilina e eosina) e processado para marcação imuno-histoquímica por anticorpos monoclonais contra as proteínas p53 e Ki-67. Os dados foram analisados pelo teste de $\chi^{2}$ para a avaliação das diferenças entre os grupos. Resultados: a idade das pacientes variou de 27 a 73 anos $(48,7 \pm 10,4$ anos). O estadiamento clínico (FIGO) foi IB1 em 27 casos (75\%) e IB2 em 9 casos (25\%). A expressão tumoral da proteína p53 foi positiva em metade dos casos. Em relação à expressão do Ki-67, foi evidenciado alto grau de proliferação celular em 73,3\% dos casos. Não houve associação da expressão das proteínas p53 e Ki-67 no tumor com idade ( $\mathrm{p}=0,091$ e 0,900$)$, estadiamento ( $\mathrm{p}=0,054$ e 0,667), tipo histológico ( $\mathrm{p}=0,674$ e 0,674), grau de diferenciação ( $\mathrm{p}=0,07$ e 0,282 ), presença de invasão linfovascular ( $\mathrm{p}=0,248$ e 0,667$)$, acometimento parametrial ( $\mathrm{p}=0,729$ e 0,763$)$ e metástases para os linfonodos pélvicos $(\mathrm{p}=0,729$ e 0,636 , respectivamente). Conclusões: a expressão tumoral das proteínas p53 e Ki-67 não se associou com achados clínico-patológicos em pacientes com carcinoma invasor do colo uterino estádio IB.

PALAVRAS-CHAVE: Neoplasias do colo uterino; Oncogenes; Proteina p53; Antígeno Ki-67

\section{ABSTRACT}

Purpose: to evaluate the association between p53 and Ki-67 expression in the tumor and clinicopathological features in patients with carcinoma of the cervix. Methods: samples were taken from the tumor of 36 patients with stage IB (FIGO) cervical carcinoma submitted to radical hysterectomy. Tissue samples were taken from the tumor, fixed in formalin and embedded in paraffin. The specimens were analyzed by histopathology (hematoxylin and eosin) and immunohistochemically evaluated using monoclonal antibodies for p53 and Ki-67. Data were analyzed statistically by the $\chi^{2}$ test to evaluate eventual differences between the groups. Results: the age of the patients ranged from 27 to 73 years ( $48.7 \pm 10.4$ years). Clinical stage (FIGO) was IB1 in 27 cases (75\%) and IB2 in 9 cases (25\%). A positive tumoral expression of the p53 protein was found in half of the cases. In relation to the Ki-67 expression, a high cell proliferation index was shown in 73.3\% of the cases. There was no association between tumoral p53 and Ki-67 expression with age ( $\mathrm{p}=0.091$ and 0.900$)$, clinical stage $(\mathrm{p}=0.054$ and 0.667$)$, histological classification $(\mathrm{p}=0.674$ and 0.674$)$, grade of differentiation $(\mathrm{p}=0.070$ and 0.282$)$, presence of lymphatic vascular space invasion ( $\mathrm{p}=0.248$ and 0.667 ), parametrial involvement ( $\mathrm{p}=0.729$ and 0.763 ) and pelvic lymph node metastasis ( $\mathrm{p}=0.729$ and 0.636 , respectively). Conclusions: tumoral expression of $\mathrm{p} 53$ and $\mathrm{Ki}-67$ was not associated with the clinicopathological features in patients with stage IB carcinoma of the cervix.

KEYWORDS: Cervix neoplasms; Oncogenes; Protein p53; Ki-67 antigen

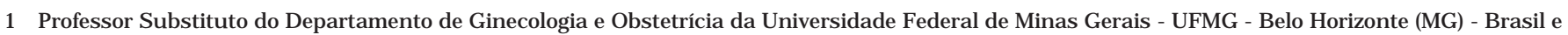
Professor da Pós-graduação em Ginecologia da Universidade Estadual Paulista “Julio Mesquita Filho” - UNESP, Botucatu (SP) - Brasil

2 Acadêmico de Medicina da Universidade Federal de Minas Gerais - UFMG - Belo Horizonte (MG) - Brasil

3 Médico do Hospital das Clínicas da Universidade Federal de Minas Gerais - UFMG - Belo Horizonte (MG) - Brasil

4 Professor do Departamento de Ginecologia e Obstetrícia da Universidade Estadual Paulista "Julio Mesquita Filho" - UNESP, Botucatu (SP) - Brasil

5,6 Professor do Departamento de Ginecologia e Obstetrícia da Universidade Federal de Minas Gerais - UFMG - Belo Horizonte (MG) - Brasil

Correspondência: Agnaldo Lopes da Silva Filho

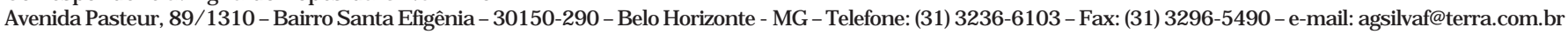


Introdução

O carcinoma de colo uterino representa grande problema de saúde pública mundial, com incidência anual de 471.000 casos e 233.000 mortes $^{1}$. A maior parte dos casos ocorre nos países em desenvolvimento devido à precariedade dos programas de rastreamento ${ }^{2}$.

O processo de carcinogênese implica, dentre outros fatores, a aquisição de alelos mutantes de genes supressores de tumor e aumento na proliferação celular ${ }^{3}$. Em decorrência dessas alterações, funções celulares que, normalmente, estariam sob controle, passam a ser exercidas de maneira desordenada, resultando na expressão exagerada de algumas proteínas responsáveis pela proliferação celular ${ }^{4}$. Entre essas proteínas encontram-se a p53, cuja função é controlar o ciclo celular, e a proteína Ki-67, utilizada como marcador de proliferação celular.

As oncoproteínas E6 e E7 do papiloma-vírus humano (HPV) de alto risco desregulam a proliferação celular 5 . A oncoproteína E6 é capaz de ligarse à proteína p53, por meio da proteína E6AP, formando o complexo p53-E6AP-E6, que é rapidamente inativado por intermédio da via proteolítica dependente da ubiquitina. Em conseqüência, não ocorre parada do ciclo para o reparo do DNA e o ciclo celular prossegue com o genoma do HPV integrado ao do hospedeiro ${ }^{5}$. Apesar de controverso na literatura, parece haver associação do gene p53, assim como da proteína p53, com a neoplasia intra-epitelial cervical (NIC) e o carcinoma invasor do colo uterino ${ }^{6}$.

O controle da divisão celular é evento crítico para o funcionamento normal das células ${ }^{4}$. Os processos reguladores da carcinogênese interferem diretamente ou indiretamente na proliferação celular ${ }^{3}$. As células em proliferação expressam em seus núcleos uma proteína não-histona de meia-vida curta, chamada Ki-67. Essa proteína está presente em todos os estágios do ciclo celular, exceto na fase $\mathrm{GO}^{7}$. A infecção por HPV 16 e 18 está associada a aumento na proliferação celular tanto em pacientes com NIC quanto no carcinoma invasor do colo uterino ${ }^{8}$.

O prognóstico da paciente com câncer de colo uterino depende de vários fatores, dentre eles, a idade, o estadiamento, o volume tumoral, o tipo histológico, a invasão do corpo uterino, a profundidade da invasão estromal, o grau de diferenciação, a invasão linfovascular e a presença de metástases linfonodais ${ }^{9}$. Por atuarem em importantes etapas da carcinogênese, é possivel que as proteínas p53 e Ki-67 possam ser utilizadas como indicadores de prognóstico para pacientes com câncer de $\operatorname{colo}^{10}$. Este estudo tem por objetivo a análise da associação da expressão das proteínas p53 e Ki-67 no tumor com achados clínico-patológicos em pacientes com carcinoma invasor de colo uterino.

\section{Métodos}

O estudo foi realizado prospectivamente em 36 mulheres com câncer do colo uterino, atendidas no Hospital das Clínicas da Universidade Federal de Minas Gerais (UFMG), no período de novembro de 2001 a setembro de 2002 . O estudo foi aprovado pelo Comitê de Ética e Pesquisa do Hospital das Clínicas da UFMG. Foram incluídas pacientes com diagnóstico de carcinoma invasor do colo uterino estádio IB de acordo com a Federação Internacional de Ginecologia e Obstetrícia (FIGO), com indicação de histerectomia radical, não submetidas à quimioterapia previamente à cirurgia .

O estadiamento do câncer de colo foi realizado de acordo com as recomendações da FIGO, por meio de exame pélvico bimanual no pré-operatório imediato logo após a anestesia ${ }^{4}$. As pacientes foram submetidas a laparotomia mediana, inventário da cavidade peritoneal, linfadenectomia pélvica e histerectomia radical, conforme a técnica de Piver et al. ${ }^{11}$.

Foi ressecado um fragmento com $1,0 \times 1,0$ $\mathrm{cm}$ do tumor, preferencialmente na sua periferia, da peça cirúrgica de histerectomia radical para avaliação imuno-histoquímica. O estudo histológico foi realizado em lâminas coradas com hematoxilina e eosina, sendo analisados o tipo histológico, o grau de diferenciação tumoral, presença de invasão linfovascular, acometimento parametrial e metástases para os linfonodos pélvicos.

Cortes histológicos seriados de cada caso, com espessura de 4 a $5 \mu \mathrm{m}$, foram obtidos em número suficiente para os anticorpos utilizados. Os cortes histológicos foram depositados sobre lâminas cobertas com adesivo poli-L-lisina (Sigma Chemical Company, St. Louis, MO, USA) e aquecidas a $60^{\circ} \mathrm{C}$, por pelo menos 12 horas. A seguir foi empregado o sistema avidina-biotinaperoxidase, descrito por Hsu et al. ${ }^{12}$, com pequenas modificações. A expressão da proteína p53 e o índice de proliferação celular (Ki-67) foram avaliados em cortes histológicos pela técnica de imunohistoquímica (IHQ) nas amostras de tumor. Os anticorpos monoclonais empregados foram o antip53 (clone DO7, DAKO Corp., Carpinteria, CA, USA) 
contra a proteína p53 e o MIB-1 (clone MIB-1, DAKO Corp.) contra o antígeno nuclear presente em fase de proliferação.

As reações foram avaliadas no mesmo microscópio óptico, com 200 vezes de aumento, na mesma intensidade de luz. A análise foi feita por patologista não informado sobre o curso clínico da paciente. Para os marcadores pesquisados, foi feita análise semiquantitativa como preconizado por Grace et al. ${ }^{13}$. Foi estabelecido um percentual de expressão protéica dividindo os tumores em categorias. Para a proteína p53, foi considerada uma expressão positiva quando ocorria a marcação IHQ em pelo menos $10 \%$ do total de células, e negativa se a porcentagem de células corada fosse menor que $10 \%$. Para a proteína Ki-67, considerou-se um tumor com alto índice de proliferação quando a marcação pela IHQ ocorria em pelo menos $25 \%$ das células e o tumor com menos de $25 \%$ das células coradas para o Ki-67 foi considerado como de baixo índice de proliferação. Essa quantificação foi realizada pelo estudo de um mínimo de 1000 células epiteliais, examinadas em cerca de 10 campos com 200 vezes de aumento. Na avaliação das proteínas p53 e Ki-67, a presença e a intensidade da coloração nuclear das células tumorais foram consideradas para quantificação.

As fichas preenchidas foram revisadas, tendo as suas informações codificadas e digitadas em banco de dados no programa SPSS para Windows versão 10.0 (SPSS Inc., Chicago, IL, USA), que desenvolveu os cálculos posteriores. Os dados foram analisados usando-se o teste de $\chi^{2}$. As diferenças ou associações com valor de $\mathrm{p}<0,05$ foram consideradas significativas.

\section{Resultados}

O estudo incluiu pacientes com idades variando entre 27 e 73 anos (média \pm desvio padrão de $48,7 \pm 10,4$ anos). A paridade média foi de $5,1 \pm 3,5$ (variação: 1 a 14) partos.

O estadiamento clínico (FIGO) foi IB1 em 27 casos (75\%) e IB2 em 9 casos (25\%). O tipo histológico era carcinoma de células escamosas (CCE) em 29 pacientes $(80,6 \%)$ e adenocarcinoma em 7 pacientes $(19,4 \%)$. O tumor era bem diferenciado (G1) em 4 (11,1\%), moderadamente diferenciado (G2) em $25(69,4 \%)$ e mal diferenciado (G3) em 7 $(19,4 \%)$ pacientes. A presença de invasão linfovascular foi observada em 9 pacientes (25\%). $\mathrm{O}$ número médio de linfonodos pélvicos dissecados foi $18,7 \pm 6,5$ (variação: 10 a 38 linfonodos). Acometimento parametrial foi evidenciado em 13 pacientes $(36,1 \%)$. A presença de metástases em linfonodos pélvicos foi observada em 13 pacientes $(36,1 \%)$, com número médio de linfonodos acometidos ao exame histológico de 1,2 $\pm 2,2$ (variação: 0 a 9 linfonodos).

A expressão tumoral da proteína p53 foi positiva ( $\geq 10 \%$ das células) em 18 pacientes (50\%). Foi evidenciado alto índice de proliferação (expressão da proteína $\mathrm{Ki}-67$ em mais de $25 \%$ das células) em 26 pacientes $(72,2 \%)$. Não houve associação entre a expressão das proteínas p53 e Ki-67 no tumor com a idade $(\mathrm{p}=0,091$ e 0,900), estadiamento $(\mathrm{p}=0,054 \mathrm{e}$ $0,667)$, tipo histológico ( $\mathrm{p}=0,674$ e 0,674$)$, grau de diferenciação ( $p=0,07$ e 0,282), presença de invasão linfovascular ( $\mathrm{p}=0,248$ e 0,667), acometimento parametrial $(\mathrm{p}=0,729$ e 0,763$)$ e presença de metástases para os linfonodos pélvicos ( $\mathrm{p}=0,729$ e 0,636) (Tabelas 1 e 2).

Tabela 1 - Associação entre idade, estadiamento, tipo histológico, grau de diferenciação, presença de invasão linfovascular, acometimento parametrial e metástases para linfonodos pélvicos com a expressão tumoral da proteína p53 em pacientes com carcinoma invasor do colo uterino.

\begin{tabular}{|c|c|c|c|}
\hline & \multicolumn{3}{|c|}{ Expressão da proteína p53 } \\
\hline & & Positiva & \multirow{3}{*}{$\mathrm{p}$} \\
\hline & \multicolumn{2}{|c|}{ ( $<10 \%$ das células) ( $\geq 10 \%$ das células) } & \\
\hline & n (\%) & n (\%) & \\
\hline Idade & & $10(66,7)$ & 0,091 \\
\hline$<40$ anos & $5(33,3)$ & $8(38,1)$ & \\
\hline$>40$ anos & $13(61,9)$ & & \\
\hline Estadiamento & & $11(40,7)$ & 0,054 \\
\hline IB1 & $16(59,3)$ & $7(77,8)$ & \\
\hline IB2 & $2(22,2)$ & & \\
\hline Tipo histológico & & $15(51,7)$ & 0,674 \\
\hline CCE & $14(48,3)$ & $3(42,9)$ & \\
\hline Adenocarcinoma & $4(57,1)$ & & \\
\hline Grau de diferenciação & & $4(100)$ & 0,070 \\
\hline G1 & $0(0)$ & $12(48)$ & \\
\hline G2 & $13(52)$ & $2(28,6)$ & \\
\hline G3 & $5(71,4)$ & & \\
\hline Invasão linfovascular & & $15(56)$ & 0,248 \\
\hline Ausente & $12(44)$ & $3(33,3)$ & \\
\hline Presente & $6(66,7)$ & & \\
\hline $\begin{array}{l}\text { Acometimento } \\
\text { parametrial }\end{array}$ & & $11(47,8 \%)$ & 0,729 \\
\hline Ausente & $12(52,2 \%)$ & $7(53,8 \%)$ & \\
\hline Presente & $6(46,2 \%)$ & & \\
\hline Metástase para & & & \\
\hline linfonodos pélvicos & & $11(47,8 \%)$ & 0,729 \\
\hline Ausente & $12(52,2 \%)$ & $7(53,8 \%)$ & \\
\hline Presente & $6(46,2 \%)$ & & \\
\hline
\end{tabular}

Nota: os valores de $\mathrm{p}$ foram calculados pelo teste do $\chi^{2}$. Negativa: expressão da proteína p53 em menos de 10\% das células; positiva: expressão da proteína p53 em mais de $10 \%$ das células (avaliação de um mínimo de 1000 células epiteliais; apenas a coloração nuclear foi considerada na quantificação da proteína p53). 
Tabela 2 - Associação da idade, estadiamento, tipo histológico, grau de diferenciação, presença de invasão linfovascular, acometimento parametrial e metástases para linfonodos pélvicos com a expressão tumoral da proteína Ki-67 em pacientes com carcinoma invasor do colo uterino.

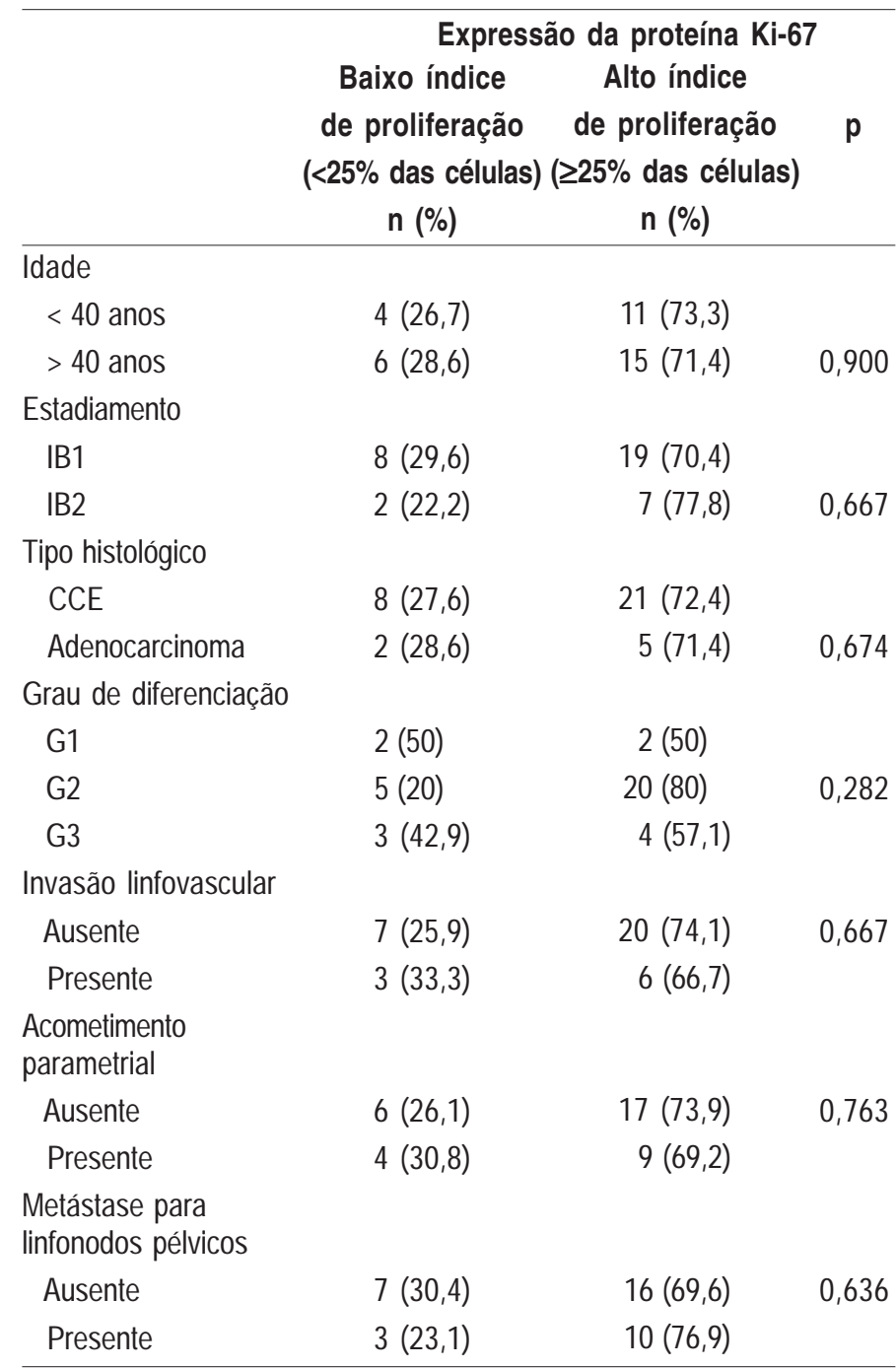

Nota: os valores de $p$ foram calculados pelo teste do $\chi^{2}$. Baixo índice de proliferação: expressão da proteína Ki-67 em menos de 25\% das células; alto índice de proliferação: expressão da proteína Ki-67 em mais de 25\% das células (avaliação de um mínimo de 1000 células epiteliais; apenas a coloração nuclear foi considerada na quantificação da proteína Ki-67).

\section{Discussão}

Neste estudo foram avaliadas as expressões das proteínas p53 e Ki-67 no tumor de pacientes com carcinoma invasor do colo uterino, visando determinar possiveis fatores prognósticos. Sabe-se que a carcinogênese é processo complexo e a avaliação de 4700 genes pela técnica do microarray mostrou 74 genes com expressão alterada em amostras de câncer do colo uterino comparadas a células normais ${ }^{14}$. Apesar disso, a identificação de novos marcadores tumorais pode ser valiosa no seguimento das pacientes, na definição daquelas que se beneficiariam de terapia adjuvante ou de tratamento primário mais agressivo ${ }^{15}$.
Alguns estudos sugerem que a expressão da proteína p53 e o aumento da atividade mitótica são eventos progressivos no processo de carcinogênese do colo uterino. Um estudo mostrou que o colo uterino normal, o condiloma acuminado, a NIC I e a NIC II apresentam expressão negativa da proteína p53, ao passo que $15 \%$ das NIC III, $29 \%$ dos CCE invasores e $11 \%$ dos adenocarcinomas apresentam expressão positiva dessa proteína ${ }^{16}$. Outro estudo mostrou expressão positiva da proteína p53 em todas as amostras de NIC III e carcinoma invasor do colo uterino e em apenas 30\% das amostras de NIC $\mathrm{II}^{13}$. Ao contrário, um estudo retrospectivo evidenciou expressão mais freqüente da proteína p53 na NIC I em comparação a NIC II e III ${ }^{17}$. Estudos avaliando amostras de colo uterino normal, NIC e carcinoma invasor mostram aumento progressivo da proliferação celular avaliada pela IHQ para Ki-67, à medida que os tecidos apresentavam alterações histológicas mais significativas ${ }^{8,18}$.

O valor da expressão protéica do p53 como fator prognóstico no carcinoma invasor do colo uterino é controversa. A presença de uma superexpressão dessa proteína é evidenciada em 20 a $82 \%$ das neoplasias invasoras do colo uterino $^{19,20}$. No presente estudo, em metade das pacientes foi identificada expressão da proteína p53 em mais de $10 \%$ das células tumorais. Não houve associação entre a expressão do p53 no tumor e a idade, estadiamento, tipo histológico, grau de diferenciação, a presença de invasão linfovascular, acometimento parametrial e metástase para linfonodos pélvicos. Um estudo envolvendo 109 pacientes com carcinoma do colo uterino, estádios IB a IIA, mostrou superexpressão da proteína p53 em $20 \%$ delas, sem associação com o prognóstico ${ }^{21}$. Outro estudo, que incluiu 52 mulheres com CCE, nos estádios I a IV, mostrou $82 \%$ de positividade para a proteína p53. Aquelas com expressão positiva apresentaram pior prognóstico ${ }^{22}$.

$\mathrm{O}$ presente estudo não mostrou correlação da expressão da proteína Ki-67 no tumor com os achados clínico-patológicos analisados. A associação da proliferação celular com achados histopatológicos, prognóstico e resposta à radioterapia em pacientes com câncer de colo foi descrita na literatura ${ }^{23-25}$. Identificou-se correlação significativa entre a expressão da proteína Ki-67 com o tamanho tumoral, disseminação linfática e sobrevida livre de doença, mas não com o grau de diferenciação tumoral ${ }^{23}$. Outro estudo retrospectivo evidenciou menor sobrevida nas pacientes com maior expressão tumoral de Ki-67 ${ }^{24}$. Não houve, porém, correlação da expressão do Ki-67 com a idade, com o tamanho tumoral, com o tipo histológico e com a presença de invasão linfovascular ou metástases linfonodais ${ }^{24}$. 
Os dados de nosso estudo não mostraram associação entre a expressão tumoral das proteínas p53 e Ki-67 com os achados clínico-patológicos em pacientes com carcinoma invasor do colo uterino estádio IB. O desenvolvimento de novas técnicas, como o microarray, propiciará o estudo de outras proteínas envolvidas no processo de carcinogênese do colo uterino que poderiam ser utilizadas como marcadores prognósticos.

\section{Referências}

1. Parkin DM, Bray FI, Devesa SS. Cancer burden in the year 2000. The global picture. Eur J Cancer. 2001;37 Suppl 8:S4-66.

2. Waggoner SE. Cervical cancer. Lancet. 2003;361(9376): 2217-25.

3. Hahn WC, Weinberg RA. Rules for making human tumor cells. N Engl J Med. 2002;347(20):1593-603.

4. Stewart BW, Kleihues P, editors. World cancer report. Lyon: IARC Press; 2003.

5. Stanley MA. Human papillomavirus and cervical carcinogenesis. Best Pract Res Clin Obstet Gynaecol. 2001;15(5):663-76.

6. Jee SH, Won SY, Yun JE, Lee JE, Park JS, Ji SS. Polymorphism p53 codon-72 and invasive cervical cancer: a meta-analysis. Int $\mathrm{J}$ Gynaecol Obstet. 2004;85(3):301-8.

7. Dabbs DJ. Diagnostic immunohistochemistry. Philadelphia: Churchill Livingstone; 2002.

8. Nair P, Nair KM, Jayaprakash PG, Pillai MR. Decreased programmed cell death in the uterine cervix associated with high risk human papillomavirus infection. Pathol Oncol Res. 1999;5(2):95-103.

9. Silva-Filho AL, Reis FM, Traiman P, Pedrosa MS, Miranda D, Triginelli SA. Clinicopathological features influencing pelvic lymph node metastasis and vaginal and parametrial involvement in patients with carcinoma of the cervix. Gynecol Obstet Invest. 2005;59(2):92-6.

10. Silva-Filho AL, Traiman P, Triginelli SA, Reis FM, Pedrosa MS, Miranda D, et al. Expression of p53, $\mathrm{Ki}-67$, and CD31 in the vaginal margins of radical hysterectomy in patients with stage IB carcinoma of the cervix. Gynecol Oncol. 2004;95(3):646-54.

11.Piver MS, Rutledge F, Smith JP. Five classes of extended hysterectomy of women with cervical cancer. Obstet Gynecol. 1974;44(2):265-72.

12. Hsu SM, Raine L, Fanger H. Use of avidin-biotinperoxidase complex $(\mathrm{ABC})$ in immunoperoxidase techniques: a comparison between $\mathrm{ABC}$ and unlabeled (PAP) procedures. J Histochem Cytochem. 1981;29(4):577-80.
13. Grace VM, Shalini JV, Sree lekha TT, Devaraj SN, Devaraj H. Co-overexpression of p53 and bcl-2 proteins in HPV-induced squamous cell carcinoma of the uterine cervix. Gynecol Oncol. 2003;91(1):51-8.

14. Ahn WS, Bae SM, Lee JM, Namkoong SE, Han SJ, Cho YL, et al. Searching for pathogenic gene functions to cervical cancer. Gynecol Oncol. 2004;93(1):41-8.

15. Geisler JP, Geisler HE. Tumor markers and molecular biological markers in gynecologic malignancies. Curr Opin Obstet Gynecol. 2001;13(1):31-9.

16. Dimitrakakis C, Kymionis G, Diakomanolis E, Papaspyrou I, Rodolakis A, Arzimanoglou I, et al. The possible role of p53 and bcl-2 expression in cervical carcinomas and their premalignant lesions. Gynecol Oncol. 2000;77(1):129-36.

17. Vassallo J, Derchain SF, Pinto GA, Martinez EZ, Syrjänen KJ, Andrade LA. High risk HPV and p53 protein expression in cervical intraepithelial neoplasia. Int J Gynaecol Obstet. 2000;71(1):45-8.

18. Keating JT, Cviko A, Riethdorf S, Riethdorf L, Guade BJ, Sun D, et al. Ki-67, cyclin E, and p16INK4 are complimentary surrogate biomarkers for human papilloma virus-related cervical neoplasia. Am J Surg Pathol. 2001;25(7):884-91.

19. Borresen AL, Helland A, Nesland J, Holm R, Trope C, Kaern J. Papillomavirus, p53, and cervical cancer. Lancet. 1992;339(8805):1350-1.

20. Skomedal H, Kristensen GB, Lie AK, Holm R. Aberrant expression of the cell cycle associated proteins TP53, MDM2, p21, p27, cdk4, cyclin D1, RB and EGFR in cervical carcinomas. Gynecol Oncol. 1999;73(2):223-8.

21.Kainz C, Kohlberger P, Gitsch G, Sliutz G, Breitenecker G, Reinthaller A. Mutant p53 in patients with invasive cervical cancer stages IB to IIB. Gynecol Oncol. 1995;57(2):212-4.

22. Nakano T, Oka K, Taniguchi N. Manganese superoxide dismutase expression correlates with p53 status and local recurrence of cervical carcinoma treated with radiation therapy. Cancer Res. 1996;56(12):2771-5.

23. Garzetti GG, Ciavattini A, Lucarini G, Goteri G, de Nictolis M, Muzzioli M, et al. MIB 1 immunostaining in stage I squamous cervical carcinoma: relationship with natural killer cell activity. Gynecol Oncol. 1995;58(1):28-33.

24. Ho DM, Hsu CY, Chiang H. MIB-1 Labeling index as a prognostic indicator for survival in patients with FIGO stage IB squamous cell carcinoma of the cervix. Gynecol Oncol. 2000;76(1):97-102.

25.Zaghloul MS, El Naggar M, Deeb A, Khaled H, Mokhtar N. Prognostic implication of apoptosis and angiogenesis in cervical uteri cancer. Int $\mathrm{J}$ Radiat Biol Phys. 2000;48(5):1409-15. 